\title{
Contributors to the 2006 IMIA Yearbook of Medical Informatics
}

\section{Editors:}

Reinhold Haux

Technical University of Braunschweig

Institute for Medical Informatics

Braunschweig, Germany

r.haux@mi.tu-bs.de

\section{Advisory Board:}

Marion Ball

IBM Center for Healthcare Management

Baltimore, MD, USA

marionball@us.bm.com

Jan H. van Bemmel

Erasmus University and

Erasmus Medical Centre

Rotterdam, The Netherlands

J.vanBemmel@ErasmusMC.nl

Alexa T. McCray

Harvard Medical School

Boston, MA,USA

amcray@bidmcharvard.edu

\section{Managing Editors:}

Elske Ammenwerth University for Health Sciences,

Medical Informatics

and Technology (UMIT)

Innsbruck, Austria

elske.ammenwerth@umit.at

Jocahim Bergmann

Technical University of Braunschweig

Insititute for Medical Informatics

Braunschweig, Germany

i.bergmann@mi.tu-bs.de

Oliver Bott

Technical University of Braunschweig

Institute for Medical Informatics

Braunschweig, Germany

o.bott@mi.tu-bs.de
Casimir Kulikowski

Rutgers - The State University of New Jersey

Dept. of Computer Science/LCSR

Piscataway, New Jersey, USA

kulikows@cs.rutgers.edu

Birgit Brigl

University of Leipzig

Insititute for Medical Informatics, Statissics and Epidemiology

Leipzig, Germany

birgit.brig|@t-online.de

Petra Knaup

University of Heidelberg

Department of Medical Informatics

Heidelberg, Germany

petra.knaup@med.uni-heidelberg.de

Elke Lang

University of Applied Sciences Darmstadt

Information and Knowledge Management

Darmstadt, Germany

lang@iuw.h-da.de

Michael Marschollek

Technical University of Braunschweig

Insititute for Medical Informatics

Braunschweig, Germany

m.marschollek@mitu-bs.de

RalfWestphal

Technical University of Braunschweig

Institute for Robotics and Process Control

Braunschweig, Germany

ralf.westphal@tu-bs.de

Simon Winkelbach

Technical University of Braunschweig

Institute for Robotics and Process Control

Braunschweig, Germany

s.winkelbach@tu-bs.de
Editorial Assistant:

Martina Hutter

Universityof Heidelberg

Medical Facully

Heidelberg, Germany

martina.hutter@med.uni-heidelberg.de

Regional Editors:

Lincoln de Assis Moura Jr.

Atech Foundation

São Paulo, Brazil

lincoln@atech.br

Don E. Detmer

President and CEO

American Medical Informatics Association

Bethesda, MD, USA

ded2x@virginia.edu

Elizabeth Di Chiara

COACH: Canada's Health Informatics Association

Toronto, ON, Canada

elizabeth@coachorg.com

RolfEngelbrecht

GSF Research Centre

Neuherberg, Germany

engel@gst.de

Arie Hasman

University of Amsterdam

Academic Medical Center

Department of Medical Informatics

Amsterdam, The Netherlands

a.hasman@amc.uva.nl

Sedick Isaacs

Department of Information Management

Groote Schuur Hospital

Observatory, South Africa

sisaacs@telkomsa.net

Yun Sik Kwak

Kyunkpook National University

Department of Medical Informatics

Daegu, Republic of South Korea

yskwak@knu.ack.

IMIA Executive Director:

Steven Huesing

Edmonton, AB, Canada

imia@shaw.ca 